\title{
Body Surface Maps in 2 Cases of Atrial Flutter
}

\author{
Seiko Kawano, M.D., Tohru Sawanobori, M.D., and \\ Masayasu Hiraoka, M.D.
}

\begin{abstract}
SUMmaRY
Body surface maps during one cycle of atrial flutter were recorded in 2 cases. The sequences of movements of the maximum and the minimum were compared to those of the sinus $P$ waves. The maps of normal sinus $P$ waves usually show that a maximum first appears at the anterior chest near the sternum and moves to the left side of the thorax in the later half of the $\mathrm{P}$ wave. The maps of atrial flutter in Case 1 showed that a maximum first appeared on the upper right back and then moved to the right side of the anterior chest. The maps of Case 2 showed that a maximum first appeared on the upper right back and then moved down the right side of the posterior thorax. There were no movements of the maxima in either case from the right side of the chest to the left as secn in the sinus $\mathrm{P}$ waves.
\end{abstract}

\section{Additional Indexing Words:}

Body surface map $\quad F$ wave Minimum

M

AJOR developments in body surface mapping techniques have evolved in the field of both clinical and experimental electrocardiology as a result of technical advances in recording and data processing. ${ }^{11-4)}$ Although this method is valuable and useful in investigating the mechanism of the activation process of the heart, there have been few reports concerning human body surface maps of atrial activation compared with those of ventricular activation. ${ }^{51-7)}$ This may be due to the problems engendered by the low voltage levels detected during atrial excitation and by the influence of respiratory changes on the actual excitation sequence. We have recently reported on body surface isopotential distribution during atrial excitation using a quantitative analysis and showing good reproducibility. ${ }^{81,91}$ Further, this method

From the Department of Cardiovascular Diseases, Medical Research Institute, Tokyo Medical and Dental University, Tokyo, Japan.

Address for reprint: Dr. Masayasu Hiraoka, Associate Professor of Clinical Physiology, Department of Cardiovascular Diseases, MRI, Tokyo Medical and Dental University, 1-5-45 Yushima, Bunkyo-ku, Tokyo 113, Japan.

Received for publication April 27, 1983. 
was applied to a single beat analysis of the $P$ wave which could be suitable for the study of excitation sequences of atrial arrhythmias.

In this study we recorded body surface maps during atrial flutter in 2 patients and compared their activation sequences with those of normal sinus $\mathrm{P}$ waves.

\section{Subjects ANd Methods}

Electrocardiographic signals were recorded simultaneously from 87 points of the body surface and from the 6 standard limb leads during the atrial activation of a single beat. We used a body surface mapper (Type 5100, Chunichi Denshi Kogyo Co, Nagoya). The methods of electrode placement and recording techniques were the same as those reported by Yamada and described in a previous report. ${ }^{9}$ ' In short, the recorded signals were stored into the computer system of the mapper and the amplitudes of electrical signals from each recorded point were drawn as matrixes of data at intervals of every 2 or $4 \mathrm{msec}$ during atrial activation. Atrial activation maps were obtained by drawing isopotential lines at $50 \mu \mathrm{V}$ intervals. F wave maps were taken during one cycle of the flutter wave which was not superimposed on a QRS and ST-T wave. We were not able to determine the zero level during flutter because the base line was always moving and never stabilized. Since these 2 patients, however, had negative $\mathrm{F}$ waves in leads II, III, and $\mathrm{aV}_{\mathrm{F}}$, we tentatively assumed the beginning of the negative deflection on the lead II ECG tracings to be the onset of the F wave. Thus, the beginning of this negative deflection was taken as zero time of the $F$ wave and analysis of the maps was done on that basis. Case 1 of atrial flutter was a 83-year-old man having a history of several years of persistent atrial flutter. He had been known to have hypertensive and ischemic heart disease with recurrent episodes of congestive heart failure for more than 5 years. Case 2 was a 39year-old female having several episodes of transient atrial flutter. She had congenital pulmonary stenosis of multiple peripheral locations, diagnosed by cardiac catheterization and angiography. The diagnosis was later confirmed by autopsy after she died suddenly 3 months after her electrocardiographic examination. Her VCG and EGG showed marked right ventricular hypertrophy and right atrial enlargement during sinus rhythm. Atrial maps during normal sinus rhythm were obtained from 22 subjects who were defined as normal based on history, physical findings, and chest roentogenograms. These were the same subjects that were reported as the normal group in previous reports. ${ }^{8), 91}$ 


\section{RESULTS}

(a) Atrial excitation maps during the sinus $\mathrm{P}$ wave

As we described in previous reports, a maximum initially appeared over the central region of the anterior chest and later moved toward the left and the back. ${ }^{8), 9)}$ The time of the initial appearance of the maximum on the map was $17.9 \pm 13.9$ msec (mean \pm SD) from the beginning of the $\mathrm{P}$ wave in the normal group. We divided the maximum into two portions, identifying them as the anterior maximum and the left maximum, depending on location relative to the left midclavicular line. In the normal group, the duration of the anterior maximum was $32 \pm 11 \mathrm{msec}$, that of the left maximum was $38 \pm$ $6 \mathrm{msec}$, and the time of shift from the anterior maximum to the left maximum after the beginning of the $\mathrm{P}$ wave was $51 \pm 12 \mathrm{msec}$.

(b) Atrial maps during atrial flutter

Fig. 1 shows the electrocardiogram and the vectorcardiogram of the $F$ loop. Fig. 2 shows the body surface atrial maps from Case 1 recorded during atrial flutter. His EGG showed prominent negative components of $F$ waves

\section{J. $\quad 83$ yrs Male}

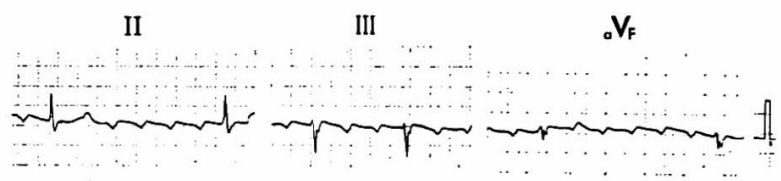

\section{F Loop}

$\mathbf{F}$

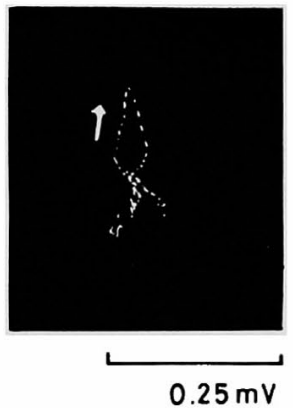

$\mathrm{S}$
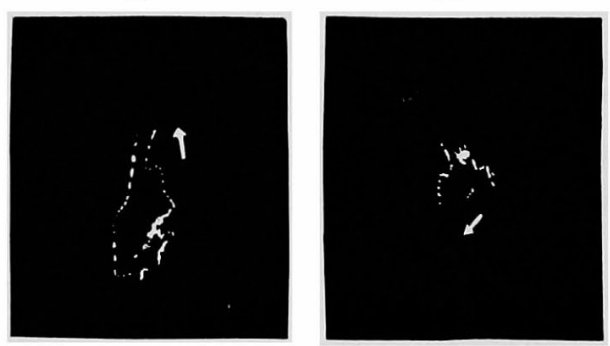

Fig. 1. ECG and VGG of F wave from Case 1, 83-year-old male.

Upper panel shows electrocardiographic tracings in leads II, III, and $\mathrm{aV}_{\mathrm{F}}$. Lower panel shows $\mathrm{F}$ loops in vectorcardiograms. Signals of F.S.H. represent frontal, left sagittal, and horizontal planes, respectively. White arrows indicate directions of the $\mathrm{F}$ loop. 

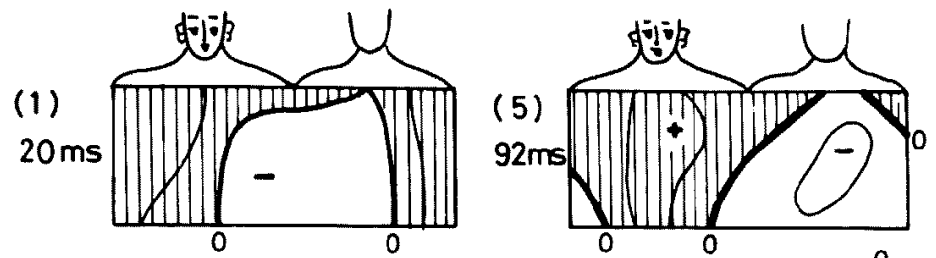

(2) $40 \mathrm{~ms}$

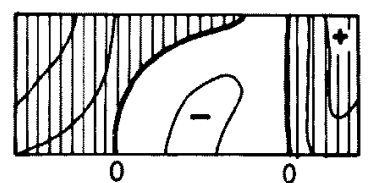

(6)

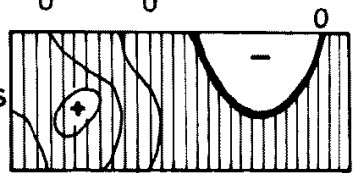

(3)
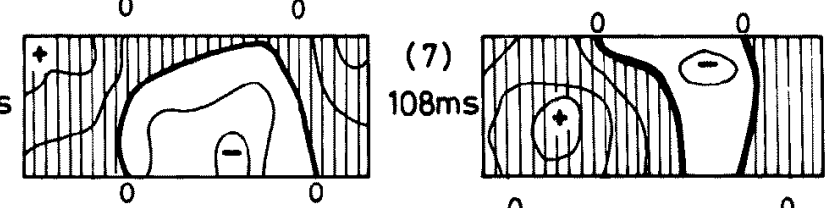

(4)
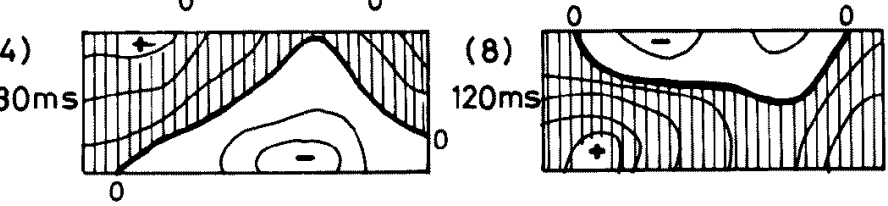

Fig. 2. Atrial maps during atrial flutter recorded from Case 1.

Number and time on the left side of each picture indicate the sequence and time after the beginning of the negative component of the $F$ wave in the lead II EGG. The striped area indicates the areas of positive potentials and the white one those of negative potentials. The symbol $(+)$ and $(-)$ indicate the maximum and the minimum, respectively. Isopotential lines are drawn at $50 \mu \mathrm{V}$ intervals. The thick line indicate a zero potential.

in leads II, III, and $a V_{F}$. F loops on the VGG showed clockwise rotation in the frontal plane and counterclockwise rotation in the left sagittal plane. On the body surface maps recorded at $20 \mathrm{msec}$ from the beginning of the $\mathrm{F}$ wave, the right side of the anterior chest was covered with positive potentials and the left side with negative potentials. A maximum could not yet be recognized at this time. A maximum first appeared on the upper right back at $40 \mathrm{msec}$ after the beginning of the $\mathrm{F}$ wave. It then moved to the right side of the shoulder at $56 \mathrm{msec}$ of the $\mathbf{F}$ wave. During this period, a minimum migrated from the left anterior chest to the left back. Then, a maximum moved down from the upper to the lower right anterior chest, and a minimum from the lower to the upper back and to the left shoulder from $80 \mathrm{msec}$ of the $F$ wave until the end of it. During one cycle of the $F$ wave, there was no change in potential from positive to negative at the anterior chest.

Fig. 3 shows the EGG and VGG recorded from case II during atrial flutter. Her ECG had a prominent negative component of the $F$ wave in leads II, III, and $\mathrm{aVF}_{\mathrm{F}}$ similar to that of Case 1. The F loop on the VCG showed counterclockwise rotation in the frontal plane and clockwise rotation 
A. Y. $\quad 39$ yrs Female

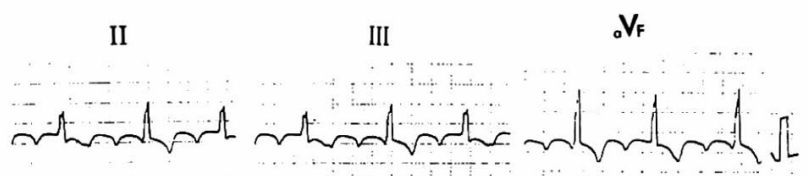

\section{F Loop}
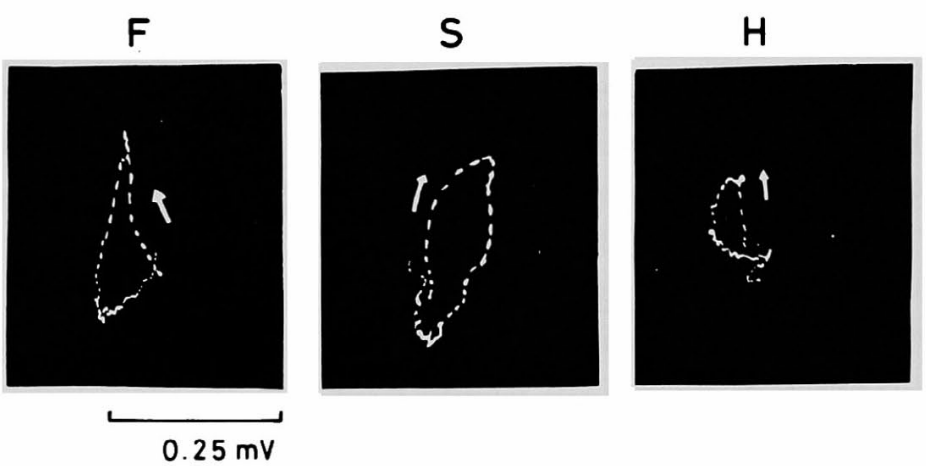

Fig. 3. ECG and VCG of F wave from Case 2, 39-year-old female. Symbols are same as those in Fig. 1.

(1) $36 \mathrm{~ms}$

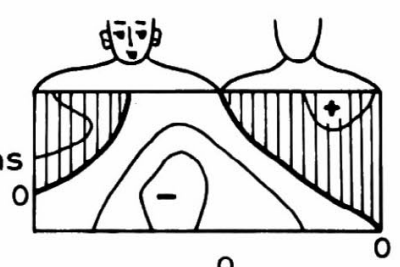

(2)

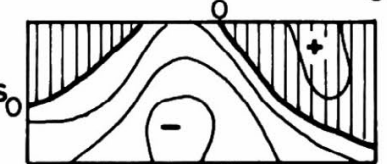

(3)

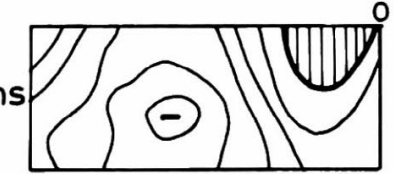

(7)

$132 \mathrm{~ms}$ $32 \mathrm{~ms}$
(5) $88 \mathrm{~ms}$
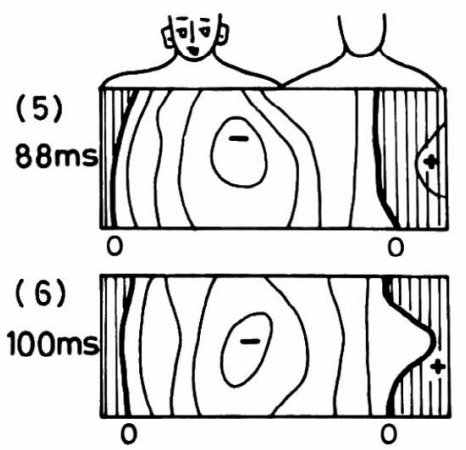

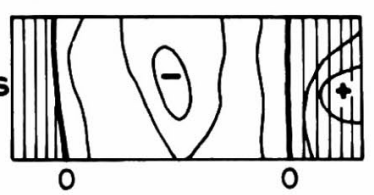

(4) $76 \mathrm{~ms}$

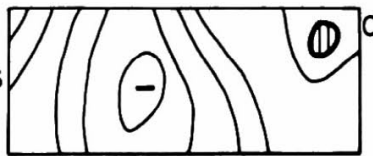

(8)
$168 \mathrm{~ms}$

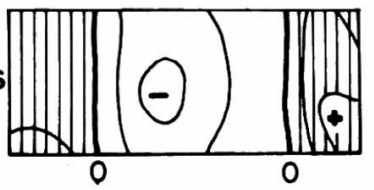

Fig. 4. Atrial maps during atrial flutter recorded from Case 2. Symbols and drawings are the same as those in Fig. 2. 
in the left sagittal plane. The body surface maps in Fig. 4 show that a maximum first appeared on the right upper back, and a minimum on the left lower chest 36 msec after the beginning of the $F$ wave. At the same time, negative potentials occupied a large portion of the anterior chest. Between 48 and 76 msec of the $F$ wave, a maximum became unrecognizable with a reduction of the positive potential area. The negative potentials covered almost all of the

\section{NORMAL}

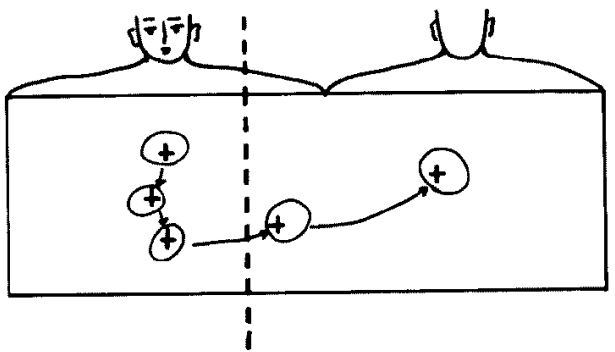

A.F.

Case 1 M. J. 83 yrs male

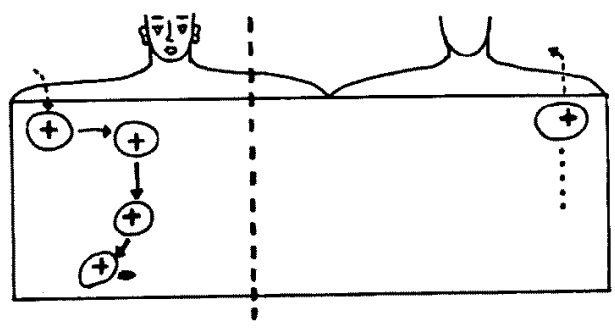

Case 2 A. Y. 39 yrs female

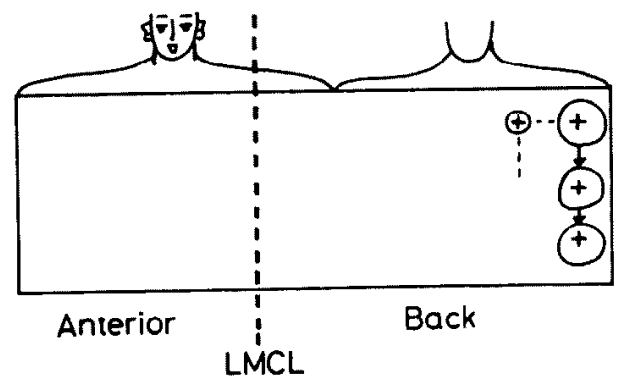

Fig. 5. Graphic schema demonstrating patterns of movement of the maximum in normal sinus $P$ wave (NORMAL) and in atrial flutter (A.F.). Symbol $(+)$ indicate the location of the maximum and arrows indicate the direction of its movement. Dashed line with arrow indicates the initial portion of the $F$ wave which was detcrmined with some uncertainty and traces the direction of its movement. LMCL: left midclavicular line. Normal pattern of sinus $\mathrm{P}$ wave is shown in the top panel. The middle panel shows the map during atrial flutter recorded from Case 1, and the bottom panel the one recorded from Case 2 . 
anterior thorax and the minimum moved upward from the lower chest to the central portion. At $88 \mathrm{msec}$ of the $\mathbf{F}$ wave, the positive potential areas began to expand again over the right lateral chest as well as over the right upper back. A maximum reappeared at the middle portion of the right back at $88 \mathrm{msec}$ of the $\mathrm{F}$ wave. At the same time, the left side of the anterior thorax was covered with negative potentials. Then, the maximum moved from the right upper back to the lower portion and the minimum moved down from the upper shoulder to the lower anterior chest until the end of the $F$ wave. In this case also there was no shift in potential from positive to negative on the anterior chest during the $F$ wave.

The body surface maps of the $F$ wave, therefore, were quite different from those of the sinus $\mathbf{P}$ wave. Fig. 5 shows the schema which demonstrates the patterns of movement of the maximum of the $F$ wave in the 2 cases of atrial flutter compared to those of the normal sinus $\mathbf{P}$ wave. As shown in the upper panel, the maximum of the sinus $P$ wave first appeared in the mid portion of the anterior thorax and then moved toward the left side of the chest. The maximum of the $\mathbf{F}$ waves in both cases of atrial flutter, however, did not show this moving pattern (Fig. 5, middle and bottom panels). Neither Case 1 nor Case 2 showed a maximum moving from the mid anterior chest toward the left lateral chest as seen with the sinus $\mathbf{P}$ wave.

\section{Discussion}

Body surface isopotential maps recorded during atrial excitation have been reported in the human as well as in the dog. ${ }^{10)-14)}$ We are, however, unaware of any report describing human body surface maps recorded during atrial flutter. In this study we succeeded in recording maps during one cycle of atrial flutter in 2 cases. There are several difficulties to obtain maps of the $F$ wave and in comparing them with those of normal sinus $P$ waves.

First, it is not possible to determine the actual beginning of the $F$ wave and its zero potential. We tentatively designated the starting point of the negative deflection of the F wave in lead II ECG as its beginning and analyzed the time-course of potential distribution on that basis. In this sense, the temporal relations of the $\mathrm{F}$ maps might not be exactly comparable to that of the sinus $\mathrm{P}$ wave. However, once the maximum and the minimum together with positive and negative potential areas became recognizable, their sequences of changes could be traced and comparison to the sinus $\mathbf{P}$ maps could be made up to the appearance of the next cycle of the $F$ wave. Secondly, there were occasional superimpositions of $F$ waves on the ST-T waves, which might have deformed the actual $\mathrm{F}$ map. We selected the $\mathrm{F}$ wave which was 
separated from the ST-T waves during a period with rather slow ventricular responses. The third problem is that a single beat analysis may not represent an actual F map of the patient. From our previous report, ${ }^{8), 91}$ however, the sinus $\mathbf{P}$ wave could be analyzed in a single beat with good accuracy and accurate reproducibility. Therefore, we believe that the maps of the $F$ wave recorded from the 2 patients in this report may well represent typical mapping patterns for them. In spite of these difficulties in analysis, we were able to demonstrate some differences between the atrial maps of the $F$ wave and the sinus $\mathbf{P}$ wave. The major differences between them are the patterns of movement of the maximum. In the $F$ wave, the maximum never moved from the right anterior chest to the left side of the thorax as usually seen in sinus $\mathrm{P}$ waves. ${ }^{8)-10), 14)}$ However, the maximum of these 2 cases appeared and migrated only from the right back to the right anterior chest in Case 1 and from the upper to the lower portion of the right back in Case 2. Another difference noted in the $F$ map was that the anterior chest potential never changed from positive to negative in either case.

As in our previous study, ${ }^{8), 9)}$ the maximum was divided into two portions depending on its location relative to the left midclavicular line. These were designated the anterior maximum when located to the right of that line and the left maximum when located to the left of that line, respectively. The former is thought to represent primarily right atrial activation and the latter left atrial activation. In this sense, the body surface maps may give us better information on the excitation sequences in individual atria than do conventional EGG and VCG. In the sinus $\mathrm{P}$ wave, the anterior maximum appeared first and, later in the $\mathrm{P}$ wave, it moved to the left side of the chest representing the left maximum. In our cases of atrial flutter, no shift of the maximum from the anterior chest to the left occurred, and further, there was no apparent left maximum recognizable in one cycle of the $F$ wave. These findings suggest several possible explanations. First the activation sequence in the $F$ wave does not move from the right atrium to the left as in the case of the sinus $P$ wave. This may indicate that the activation in major portions of the right and the left atrium occur almost simultaneously in opposite directions, thus cancelling their individual electrical forces. Another possibility is that the left atrial activation potential is too small to be recognized on the body surface due to disorganized or random excitation spread from that chamber. Alternatively, the left atrium may be electrically quiescent during the $F$ wave. Epicardial mapping during atrial flutter would probably gives us a detailed explanation of the above. There was one report describing epicardial excitation of the atria in a patient with atrial flutter. ${ }^{15)}$ Although in that report the left atrium was not sufficiently explored because of technical reasons, a 
much delayed activation of the left atrium was noted. Boineau et al examined the spread of epicardial excitation in a case of naturally occurring flutter in a dog. This resembled the ECG pattern commonly seen in human atrial flutter. ${ }^{22)}$ The epicardial activation sequence map showed early as well as delayed excitation spread and a local conduction block in the left atrium. Whether such disorganized excitation sequences in the left atrium are common findings or not during experimental as well as clinical atrial flutter await further studies with refined techniques.

The circus movement theory, which postulates the flutter wave traveling in a circuit around the venae cavae up or down the taenia terminalis, has been a widely accepted concept especially with regard to the common type of flutter that shows a continuous undulation of the base line and a prominent negative component of the $\mathrm{F}$ waves in leads II, III, and $\mathrm{aV}_{\mathrm{F} .}{ }^{16)-23)}$ Our cases represented the common type of flutter according to their ECG patterns. From the standpoint of the circus movement theory, we suggest that the findings of those body surface maps might indicate the flutter wave going up the posterior wall of the right atrium and down its anterior wall near the anterior scptum in Casc 1. In Case 2, the wavefronts traveled up the anterior septum and down the posterior wall of the right atrium based upon the locations of both atria and also the behaviour of the maximum and the minimum on the body surface map. In neither case did the left atrium form a major route of the re-entry circuit. Our body surface map findings, however, cannot exclude the possibility that an automatic focus at the atrial septum fires rapidly and exitation propagates to both atria almost simultaneously. Clarification of the mechanism of atrial flutter requires further studies.

\section{REFERENCES}

1. Taccardi B, De Amvroggi L, Vigarott $\mathrm{C}$ : Body surface mapping of heart potentials. in The Theoretical Basis of Electrocardiology, by Nelson CV, Geselowitz, Clarendon Press, Oxford, p 436, 1976

2. Spach MS, Bar RC, Blumenshein SD, Boineau JP: Clinical implications of isopotential surface maps. Ann Int Med 69: 919, 1968

3. Lepeschkin E: History of electrocardiographic thoracic mapping. Adv Cardiol 10: 2, 1974

4. Yamada K: Body surface isopotential map; past, present and future. Jpn Circulat J 45: 1,1981

5. Yamada K, Toyama J, Wada M, Sugiyama S, Sugenoya J, Toyoshima H, Mizuno Y, Sotobata I, Kobayashi T, Okajima M: Body surface isopotential mapping in Wolf-ParkinsonWhite syndrome. Noninvasive method to determine the localization of the accessory atrioventricular pathway. Am Heart J 90: 721, 1975

6. Mirvis DM, Keller FW, Ideken RE, Cox JW, Zettergen DG, Dowdie RF: Value and limitations of surface isopotential mapping techniques in the detection and localization of multiple discrete epicardial events. J Electrocardiol 10: 347, 1977

7. Flower NC, Horan LG, Sohi GS, Chreshand R, Johnso JC: New evidence for inferoposterior 
myocardial infarction on surface potential maps. Am J Cardiol 38: 576, 1976

8. Kawano S, Yamamoto M, Sawanobori T, Hiraoka M, Sakamoto $Y$ : The body surface isopotential distributions during atrial excitation in atrial disorders. Jpn Circulat J 45: 915 , 1981

9. Kawano S, Sawanobori T, Hiraoka M: Human body surface mapping during atrial depolarization in normal and diseased subjects. J Electrocardiol 16: 151, 1983

10. Taccardie B: Body surface distribution of equipotential lines during atrial depolarization and ventricular repolarization. Circulat Res 19:865, 1966

11. Spach MS, King TD, Barr RC, Boaz DE, Morrow MN, Herman-Giddens S: Electrical potential distribution surrounding the atria during depolarization and repolarization in the dog. Circulat Res 24: 857, 1969

12. King TD, Barr RC, Herman-Giddens S, Boaz DE, Spach MS: Isopotential body surface maps and their relationship to atrial potentials in the dog. Circulat Res 30: 393, 1972

13. Spach MS, Barr RC, Warren RB, Benson DW, Walson A, Edwards SB: Isopotential body surface mapping in subjects of all ages. Emphasis on low-level potentials with analysis of the method. Circulation 59: 805, 1979

14. Mirvis DM: Body surface distribution of electrical potential during atrial depolarization and repolarization. Circulation 62: 162, 1980

15. Wellence HJJ, Janse MJ, Vam Dam RT, Durrer D: Epicardial excitation of the atria in a patient with atrial flutter. Br Heart J 33: 233, 1971

16. Lewis T, Feil HS, Stroud WD: Observations upon flutter and fibrillation. Part II. The nature of auricular fibrillation. Heart 7: 191, 1920

17. Katz LN, Pick A: The mechanism of auricular flutter and auricular fibrillation. Circulation 8: 601,1953

18. Rytand DA: The circus movement (entrapped circuit wave) hypothesis and atrial flutter. Ann Int Med 65: 125, 1966

19. Kimura E, Sato M, Murao S, Ajisaka H, Koyama S, Omiya Z: Experimental studies on the mechanism of the auricular flutter. Tohoku J Exp Med 60:197, 1954

20. Moe GK, Pastelin G, Mendez R: Circus movement excitation of the atria. in Physiology of Atrial Pacemakers and Conduction Tissues, Futura Publ Co, Mount Kisco, New York, p 207, 1980

21. Pastelin G, Mendez R, Moe GK: Participation of atrial specialized conduction pathways in atrial flutter. Circulat Res 42: 386, 1977

22. Boineau JP, Schuessler RB, Moonez C, Miller CB, Wylds AC, Hudson RD, Borremans JM, Brocks CW: Natural and evoked atrial flutter due to circus movement in dogs. Am J Cardiol 45: 1167, 1980

23. Allessie MA, Bonke, FIM, Schopman FJG: Circus movement in rabbit atrial muscle as a mechanism of tachycardia. II. The role of nonuniform recovery of excitability in the occurrence of unidirectional block, as studied with multiple microelectrodes. Circulat Res 39: 168,1976 